\title{
Stress induced (Takotsubo) cardiomyopathy triggered by the COVID-19 pandemic
}

\author{
SOFIA GIANNITSI ${ }^{1}$, PAVLOS TSINIVIZOV ${ }^{1}$, LEONIDAS E. POULIMENOS ${ }^{1}$, \\ MANOLIS S. KALLISTRATOS ${ }^{1}$, DIMITRIS VARVAROUSIS ${ }^{1}$, ATHANASIOS J. MANOLIS ${ }^{1}$, \\ KONSTANTINOS TSAMAKIS ${ }^{2,3}$, EMMANOUIL RIZOS ${ }^{2}$, DEMETRIOS A. SPANDIDOS ${ }^{4}$, \\ DIMITRIOS TSIPTSIOS $^{5}$ and ANDREAS S. TRIANTAFYLLIS ${ }^{1}$
}

\author{
${ }^{1}$ Department of Cardiology, Asklepeion General Hospital, 16673 Athens; ${ }^{2}$ Second Department of Psychiatry, \\ University of Athens, 'ATTIKON' University Hospital, 12462 Athens, Greece; ${ }^{3}$ King's College London, \\ Institute of Psychiatry, Psychology and Neuroscience, London SE58AF, UK; ${ }^{4}$ Laboratory of Clinical Virology, \\ School of Medicine, University of Crete, 71003 Heraklion, Greece; ${ }^{5}$ Neurophysiology Department, \\ South Tyneside and Sunderland NHS Foundation Trust, Sunderland SR47TP, UK
}

Received June 26, 2020; Accepted July 7, 2020

DOI: $10.3892 /$ etm.2020.8968

\begin{abstract}
Stress induced (Takotsubo) cardiomyopathy (TC) represents an acute heart failure syndrome triggered by physical or emotional stressors. COVID-19 pandemic has caused an unprecedented health crisis resulting in fear, distress and anxiety, with emerging cardiovascular implications. COVID-19 related stress can act as potential trigger for TC. We present a case of an elderly female who developed TC due to stress surrounding COVID-19.
\end{abstract}

\section{Case presentation}

A 79-year old female presented with acute chest pain after watching the daily TV report on the death toll of COVID-19 pandemic. Her medical history included arterial hypertension under treatment with ace-inhibitors. She was haemodynamically stable, with a blood pressure of 130/70 $\mathrm{mmHg}$, a heart rate of 75 beats/min in sinus rhythm, and an oxygen saturation of $99 \%$ in room air. Physical examination and body temperature were normal. Electrocardiogram recorded diffuse ST segment elevation (Fig. 1A). Emergency coronary angiography excluded stenotic lesions (Fig. 1B and C). Left ventriculography illustrated severe hypokinesia in the mid-apical segments, hyperdynamic basal segments

Correspondence to: Dr Konstantinos Tsamakis, Second Department of Psychiatry, University of Athens, 'ATTIKON' University Hospital, Rimini 1, Chaidari, 12462 Athens, Greece E-mail:ktsamakis@gmail.com

Key words: Takotsubo cardiomyopathy, stressor, COVID-19, pandemic, stress
(Fig. 1D, systole; and E, diastole), and impaired left ventricular systolic function with an ejection fraction of $35 \%$. High sensitive troponine-T was elevated reaching a peak after $12 \mathrm{~h}$ $(2.950 \mathrm{pg} / \mathrm{ml}$, normal $<15.6 \mathrm{pg} / \mathrm{ml})$, whereas other routine blood tests, including inflammatory markers, were normal.

\section{The diagnosis}

Stress induced (Takotsubo) cardiomyopathy.

\section{Discussion}

Takotsubo cardiomyopathy (TC), also called stress-induced cardiomyopathy, mimics an acute coronary syndrome (ACS), predominantly affecting elderly women and is triggered by an emotional or physical stressor (1), in our case the COVID-19 death update. The term 'Takotsubo' means 'octopus pot' in Japanese and describes the pathognomonic left ventricular end-systolic apical ballooning, illustrated in transthoracic echocardiogram (TTE) and left ventriculography during cononary angiography (1).

Clinical presentation, electrocardiographic findings, and biomarker profiles are similar to those of an ACS (2). TC represents an acute heart failure syndrome with substantial morbidity and mortality (2). The in-hospital and one-year mortality of TC has been reported $1-5$ and 5.6\%, respectively, with a recurrence rate of $\leq 5 \%(1,2)$. Left ventricular systolic function usually recovers within four weeks (1). TC is characterized by a low-grade chronic inflammatory state consisting of myocardial macrophage inflammatory infiltrates, substantial increase in the pro-inflammatory, classical monocyte subset $\mathrm{CD} 14^{++} \mathrm{CD} 16^{-}$, and an increase in the systemic pro-inflammatory cytokines interleukin-6 (IL-6), IL-8 and chemocine CXCL1 (3). This localized and systemic inflammatory response plays a pivotal role in the 


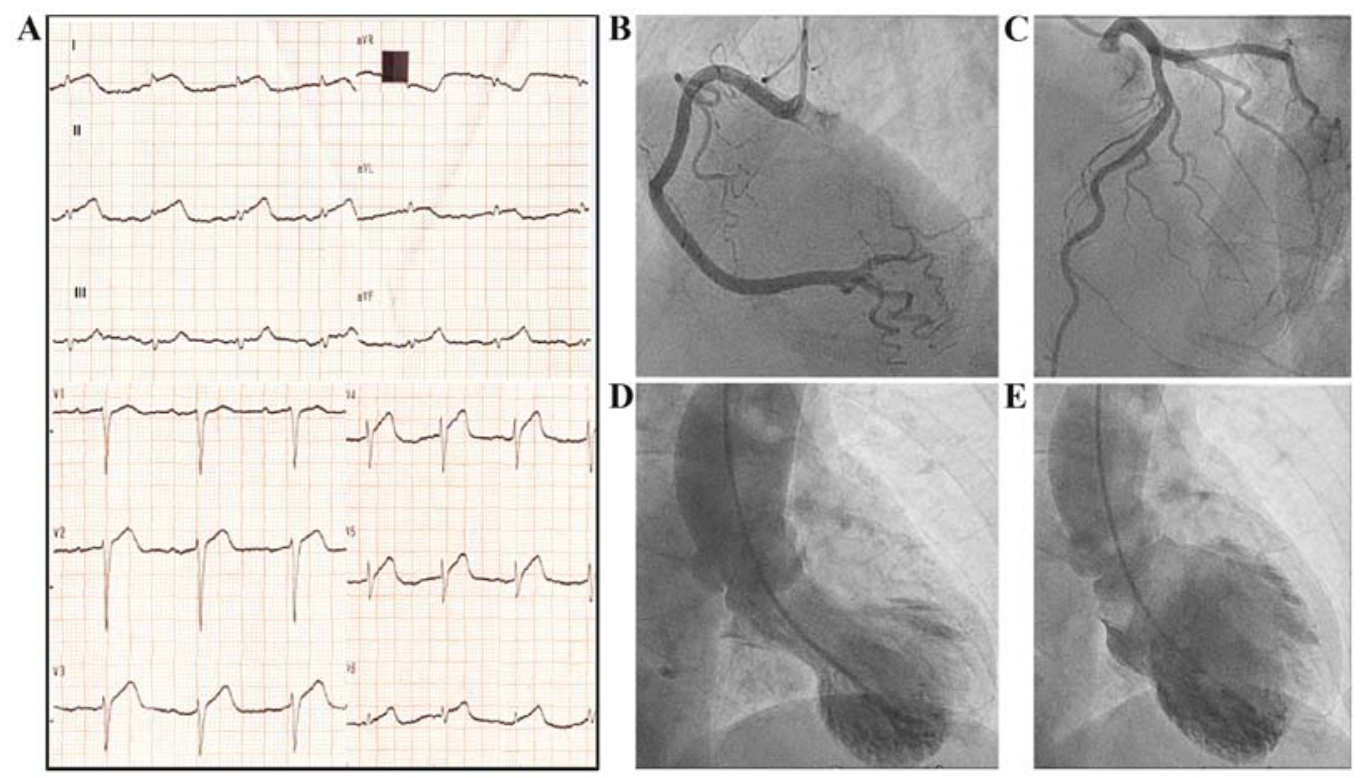

Figure 1. Electrocardiogram showing diffuse ST segment elevation (A). Emergency coronary angiography excluded stenotic lesions (B and C). Left ventriculography illustrating severe hypokinesia in the mid-apical segments and hyperdynamic basal segments (D, systole; E, diastole).

development of TC, while it might also serve as a promising therapeutic target for which no effective treatment currently exists (3).

The brain-heart interaction has been acknowledged as a significant factor in the pathogenesis of TC. Overstimulation of the sympathetic nervous system is hypothesized as the underlying mechanism $(2,4)$. The hypothalamic-pituitary-adrenal axis (HPA-axis) is a major neuroendocrine system which regulates the release of cortisol from the adrenal gland, shifting the metabolism to higher stress levels. Higher serum cortisol levels and increased sympathetic activity may cause myocardial damage (5). Consequently, acute psychological or physical stress mediated via the sympathetic-adrenalmedulla axis with catecholamine release in the adrenal medulla and the HPA axis with consecutive cortisol release from the adrenal cortex may act as a trigger for TC (5). Additionally, regional differences in myocardial expression of $\beta 2$-adrenergic receptor density have been shown, which mediate the cellular effects of the increased catecholamine blood concentrations and explain the regional left ventricular myocardial stunning (5).

Therefore, the enormous psychological strain posed on the community by the pandemic can activate the brain-heart axis and serve as a potential stressor for TC (4,6). COVID-19 pandemic has caused an unprecedented health crisis resulting in individual and societal fear and anxiety, with emerging cardiovascular implications, which we should be aware of (4).

The patient was transferred to the coronary unit where treatment with ace-inhibitor, aspirin, b-blocker and statin was initiated. Her course was uncomplicated with gradual normalization of troponin. She was discharged after one week in good condition and normal echocardiography, and was advised to avoid following news related to the pandemic.

\section{Acknowledgements}

Not applicable.

\section{Funding}

No funding was received.

\section{Availability of data and materials}

Not applicable

\section{Authors' contributions}

SG and AST wrote the original draft, edited and critically revised the manuscript. KT, DT, DV, ER, PT, LEP, MSK, DAS and AJM critically revised and edited the manuscript. All authors substantially contributed to the conception, writing and revision of the work and approved the final content of the manuscript.

\section{Ethics approval and consent to participate}

Not applicable.

\section{Consent for publication}

Not applicable.

\section{Competing interests}

DAS is the Editor-in-Chief for the journal, but had no personal involvement in the reviewing process, or any influence in terms of adjudicating on the final decision, for this article. The other authors declare that they have no competing interests.

\section{References}

1. Dagres N, Triantafyllis A and Anastasiou-Nana M: A 70 year old woman with chest pain after a stressful event. BMJ 347: f5583, 2013. 
2. Templin C, Ghadri JR, Diekmann J, Napp LC, Bataiosu DR, Jaguszewski M, Cammann VL, Sarcon A, Geyer V, Neumann CA, et al: Clinical features and outcomes of Takotsubo (Stress) cardiomyopathy. N Engl J Med 373: 929-938, 2015.

3. Scally C, Abbas H, Ahearn T, Srinivasan J, Mezincescu A, Rudd A, Spath N, Yucel-Finn A, Yuecel R, Oldroyd K, et al: Myocardial and systemic inflammation in acute stress-induced (Takotsubo) cardiomyopathy. Circulation 139: 1581-1592, 2019.

4. Tsamakis K, Triantafyllis AS, Tsiptsios D, Spartalis E, Mueller C, Tsamakis C, Chaidou S, Spandidos DA, Fotis L, Economou M and Rizos E: COVID-19 related stress exacerbates common physical and mental pathologies and affects treatment (Review). Exp Ther Med 20: 159-162, 2020
5. Buchmann SJ, Lehmann D and Stevens CE: Takotsubo cardiomyopathy-acute cardiac dysfunction associated with neurological and psychiatric disorders. Front Neurol 10: 917, 2019.

6. Tsamakis K, Rizos E, Manolis AJ, Chaidou S, Kympouropoulos S, Spartalis E, Spandidos DA, Tsiptsios D and Triantafyllis AS: COVID-19 pandemic and its impact on mental health of healthcare professionals. Exp Ther Med 19: 3451-3453, 2020.

(c) (1) (9) This work is licensed under a Creative Commons Attribution-NonCommercial-NoDerivatives 4.0 International (CC BY-NC-ND 4.0) License. 